\title{
FUNÇÃO DE AFILAMENTO PARA Virola surinamensis (ROLL.) WARB ${ }^{1}$
}

\author{
Helio Garcia Leite ${ }^{2}$, João Ricardo Vasconcellos Gama ${ }^{3}$, Jovane Pereira da Cruz ${ }^{3}$ e Agostinho Lopes de Souza ${ }^{2}$ \\ RESUMO - Este estudo foi conduzido com o propósito de gerar equação de afilamento para \\ Virola surinamensis (Roll.) Warb, que está entre as espécies mais exportadas pela indústria madeireira do estuário \\ amazônico. Foram testados os modelos de Kozak (1969), Baldwin (1991), Demaerschalk (1972) e Garay (1979). \\ Todos esses modelos estimaram o diâmetro comercial com precisão, porém os três primeiros resultaram em \\ inconsistência ao estimar a altura comercial. O modelo de Garay foi selecionado e utilizado para gerar uma \\ curva de cubagem para a espécie.
}

Palavras-chave: Várzea, manejo florestal, função de afilamento e Virola surinamensis.

\section{TAPERING FUNCTION FOR Virola surinamensis (ROLL.) WARB}

\begin{abstract}
This study was carried out with the aim of generating a taper equation for Virola surinamensis (Roll.) Warb, which is among the main exported species by the wood industries from the Amazonian estuary. The Kozak (1969), Baldwin (1991), Demaerschalk (1972) and Garay (1979) mathematical models were tested. All the tested models estimated the diameter with good precision, however, the first three models resulted in inconsistency while estimating commercial height. The Garay model was chosen and used to generate a cubage curve for the species.
\end{abstract}

Keywords: Floodplain, forest management, taper and Virola surinamensis.

\section{INTRODUÇÃO}

Na área do estuário amazônico, ao norte do Brasil, a pressão sobre os recursos da floresta tem causado redução no estoque de madeira de algumas espécies de interesse econômico. Como exemplo, tem-se a exploração da Virola surinamensis, que está entre as espécies madeireiras mais exportadas pela indústria madeireira do estuário amazônico.

Cada vez mais o mercado exige produtos diversificados e de qualidade. Como os produtos florestais não fogem à regra, faz-se necessário utilizar técnicas de quantificação e qualificação desses produtos, entre as quais se têm os modelos de afilamento que possibilitam a determinação do volume total e comercial de madeira para qualquer diâmetro ou altura especificados.
Os modelos de afilamento são relações funcionais que permitem descrever o perfil longitudinal de um tronco. São funções consideradas dinâmicas, porque possibilitam a estimativa do diâmetro (di) de dada altura (hi) e altura a qualquer diâmetro especificado, como também servem para fazer o sortimento e estudar a evolução da forma da árvore ao longo de sua vida (KOZAK et al.,1969).

A integral de um modelo de afilamento permite estimar o volume entre quaisquer segmentos ao longo do fuste - procedimento denominado sortimento - e, desse modo, maximiza-se o aproveitamento de uma árvore e, conseqüentemente, de um povoamento florestal. O estudo do sortimento de povoamentos de florestas de produção (nativas ou plantadas) é uma das ferramentas

\footnotetext{
${ }^{1}$ Recebido em $1^{\circ} .06 .2004$ e aceito para publicação em 10.11.2005.

${ }^{2}$ Professor do Departamento de Engenharia Florestal, UFV, 36570-000 Viçosa-MG.

${ }^{3}$ Programa de Pós-Graduação em Ciência Florestal da UFV, Viçosa-MG.
} 
importantes no planejamento da utilização dos recursos florestais e na avaliação do manejo empregado, em razão de permitir a quantificação da matéria-prima, classificando-a de acordo com as dimensões e qualidade, para os múltiplos usos da madeira (FISCHER, 1997).

Segundo Guimarães e Leite (1992), é uma técnica já bastante difundida nos países com maior tradição florestal, onde constituí fator importante nas decisões de manejo e planejamento florestais e, portanto, na maximização do aproveitamento da madeira produzida. A utilização de modelos de afilamento é uma conseqüência natural do desenvolvimento do setor florestal no Brasil, em que o aperfeiçoamento das técnicas de inventário e a necessidade de flexibilização da informação dos estoques de madeira despertaram maior interesse no aprimoramento das informações de inventário (RIOS,1997).

No Brasil, as pesquisas que analisam o perfil do tronco de árvores e que convertem florestas em multiprodutos da madeira são mais direcionadas para Eucalyptus e Pinus. Quanto às espécies nativas, destacam-se os trabalhos de Garcia et al. (1993) e Moura (1994). Como a madeira de virola é utilizada para a fabricação de grandes, médias e pequenas peças, o sortimento possibilitará o direcionamento do estoque conforme as alternativas mais lucrativas, sendo possível maximizar financeiramente os estoques de virola existentes nas florestas de várzea do estuário amazônico.

Nesse sentido, o presente estudo teve como objetivo avaliar a acuracidade de alguns modelos de afilamento para quantificar o sortimento de Virola surinamensis.

\section{MATERIAL E MÉTODOS}

\section{1. Área de estudo}

A área de estudo foi uma floresta não explorada de várzea baixa localizada no rio Medonho, Município de Afuá, ao norte do Estado do Pará. O clima da região é quente e úmido do tipo $\mathrm{A}_{\mathrm{mi}}$, segundo Köeppen; a precipitação média anual, de $2.500 \mathrm{~mm}$; e a temperatura média anual e a umidade relativa do ar correspondem a $26{ }^{\circ} \mathrm{C}$ e $85 \%$, respectivamente. O período mais seco vai de setembro a novembro e o mais chuvoso, de fevereiro a abril (SUDAM, 1984).

A região apresenta extensa rede hidrográfica, com topografia plana a suavemente ondulada. Os solos são originados de formação geológica do quaternário, e o material que os compõe é oriundo de sedimentos holocênicos, com elevada fertilidade natural e conteúdo de bases trocáveis variáveis; na classificação de Vieira (1988), são solos hidromórficos, gleizados e pouco húmicos.

\subsection{Informações sobre a espécie}

A virola é uma espécie da família Myristicaceae, de porte médio (até $40 \mathrm{~m}$ de altura e DAP $<1,0 \mathrm{~m}$ ), fuste monopodial e copa cuneiforme. Seus ramos apresentam folhas alternas, verdes glabras, com base obtusa e ápice acuminado. Ainflorescência é em forma de panículas axilares ou subaxilares, com pedicelos opostos lateralmente; possuí fascículos de 8 a 15 flores nas extremidades dos ramos; flores femininas raras, com ovário de forma ovóide e estilete curto; o estigma é emarginado, bífido e ereto. O fruto é em forma de elipse, com 14 a 16 mm de comprimento; curto estipilado, com frutificação de fevereiro a julho. O tronco apresenta ramificações regulares, verticilada, quase horizontal; a casca é espessa, esbranquiçada e de coloração castanha no interior (Van-ROOSMALEN et al., 1996).

A semente é recalcitrante, apresenta dispersão primária endozoocórica/barocórica e secundária hidrocórica. No Brasil, distribui-se desde a metade oriental da região amazônica, principalmente no estuário amazônico, Estados de Roraima, do Maranhão até Pernambuco, como também nas Güianas, na Venezuela, Colômbia e em Trinidad e Antilhas. É uma espécie que cresce nos solos inundáveis de várzea, com área de ocorrência natural, situando-se entre $0^{\circ}$ e $5^{\circ}$ sul (LOPES et al., 1997).

A madeira de virola é leve, com densidade em torno de $0,50 \mathrm{~g} / \mathrm{cm}^{3}$; seu cerne varia de bege-claro a castanhoescuro; alburno bem desenvolvido, sem gosto e com cheiro distinto (OLIVEIRA, 1972). É uma das espécies mais processadas nas indústrias madeireiras do estuário amazônico, por ter boa valorização econômica no mercado internacional, sendo uma das espécies preferidas para a fabricação de compensado, embalagens, artigos de esporte, brinquedos, lápis, palitos, bobinas e carretéis, entre outros utensílios (SUDAM, 1981). No período de 1980-98, mesmo apresentando taxa decrescente de comercialização devido à política de contingenciamento, a virola foi a terceira espécie arbórea mais exportada para o mercado internacional de madeiras tropicais (ANGELO et al., 2001). 


\subsection{Coleta de dados e análises}

Com a árvore em pé, foi medido o diâmetro à altura de 1,30 m (DAP), ou logo acima da sapopema, de 93 indivíduos com diâmetro variando de 14 a $80 \mathrm{~cm}$. Posteriormente, as árvores foram derrubadas, e se realizaram as mensurações da altura de cepa, fuste (parte comercial) e copa; os diâmetros foram medidos com casca em 15 secções ao longo do fuste, nos pontos correspondentes a 1, 2, 3, 4, 5, 10, 20, 30, 40, 50, 60, 70, 80, 90 e $100 \%$ da altura de fuste. Para a obtenção do volume individual, utilizou-se o método de Smalian. Foram testados os modelos, seguidos pelas respectivas expressões de cálculo de d, h e V:

a) Kozak et al. (1969)

$$
\begin{aligned}
& d^{2} \operatorname{dap}^{-2}=\beta_{0}+\beta_{1}\left(\frac{h}{H t}\right)+\beta_{2}\left(\frac{h}{H t}\right)^{2}+\varepsilon \\
& d=\operatorname{dap} \sqrt{\beta_{0}+\beta_{1}\left(\frac{h}{H t}\right)+\beta_{2}\left(\frac{h}{H t}\right)^{2}} \\
& h=\left(-\beta_{1} H t \pm \sqrt{\left(\beta_{1} H t\right)^{2}-4 \beta_{2}\left(\beta_{0} H t^{2}-d^{2} H t^{2} d a p^{-2}\right)}\right)^{-2 \beta_{2}} \\
& V=\operatorname{Kdap}^{2}\left(\beta_{0}\left(h_{2}-h_{1}\right)+\frac{\beta_{1}}{2 H t}\left(h_{2}{ }^{2}-h_{1}{ }^{2}\right)+\frac{\beta_{2}}{3 H t^{2}}\left(h_{2}{ }^{3}-h_{1}{ }^{3}\right)\right)
\end{aligned}
$$

b) Demaerschalk (1972)

$$
\begin{aligned}
& d^{2} \operatorname{dap}^{-2}=10^{(2 \beta 0)} d a p^{(2 \beta 1-2)} h t^{(2 \beta 2)}(h t-h)^{(2 \beta 3)}+\varepsilon \\
& d=10^{\beta_{0}} d a p^{\beta_{1}} H t^{2 \beta_{2}}(H t-h)^{2 \beta_{3}} \\
& h=H t-\left(d\left(10^{\beta_{0}} d a p^{\beta_{1}} H t^{\beta_{2}}\right)^{-1}\right)^{1 / \beta_{3}} \\
& V=K 10^{2 \beta_{0}} \operatorname{dap}^{2 \beta_{1}} H t^{2 \beta_{2}}\left(\left(H t-h_{1}\right)^{2 \beta_{3}+1}-\left(H t-h_{2}\right)^{2 \beta_{3}+1}\right)^{-2 \beta_{3}-1}
\end{aligned}
$$

c) Baldwin (1991)

$$
\begin{aligned}
& d d a p^{-1}=\left(\beta_{0}+\beta_{1} \operatorname{Ln}\left(1-t\left(h^{1 / 3} H t^{-1 / 3}\right)\right)+\varepsilon\right. \\
& d=\operatorname{dap}\left(\beta_{0}+\beta_{1} \operatorname{Ln}\left(1-t\left(h^{1 / 3} H t^{-1 / 3}\right)\right)\right. \\
& h=H t\left(\left(1-\exp \left(\left(d d a p^{-1}\right)-\beta_{0}\right) \beta_{1}^{-1}\right) t^{-1}\right)^{3} \\
& V=\operatorname{Kdap}^{2} H t\left(\begin{array}{l}
\beta_{0}^{2}\left(h_{2}-h_{1}\right) H t^{-1}+2 \beta_{0} \beta_{1} 3 t^{-3}(a(p x-q x \operatorname{Ln} a)-b(p y-q y \operatorname{Ln} b)) \\
-\beta_{1}^{2} 3 t^{-3}\left(a q x \operatorname{Ln}^{2} a-2 a p x \operatorname{Ln} a+2 a r x-b q y \operatorname{Ln}^{2} b+2 b p y \operatorname{Ln} b-2 b r y\right)
\end{array}\right) \\
& \text { d) Garay (1979) }
\end{aligned}
$$$$
\begin{aligned}
& d \operatorname{dap}^{-1}=\beta_{0}\left(1+\beta_{1} \operatorname{Ln}\left(1-\beta_{2} h^{\beta_{3} H t}{ }^{-\beta_{3}}\right)\right)+\varepsilon \\
& d=\operatorname{dap} \beta_{0}\left(1+\beta_{1} \operatorname{Ln}\left(1-\beta_{2} h^{\beta_{3}} H^{-\beta_{3}}\right)\right) \\
& h=H t\left(\left(-\exp \left(\left(d-\operatorname{dap} \beta_{0}\right)\left(\operatorname{dap} \beta_{0} \beta_{1}\right)^{-1}\right)-1\right) \beta_{2}{ }^{-1}\right)^{1 / \beta_{3}}
\end{aligned}
$$

em que:

$$
\begin{array}{lll}
K=\pi / 40000 & p x=1-a / 2+a^{2} / 9 & r x=1-a / 4+a^{2} / 27 \\
t=1-\exp \left(-\beta_{0} \beta_{1}^{-1}\right) & p y=1-b / 2+b^{2} / 9 & r y=1-b / 4+b^{2} / 27 \\
a=1-t\left(-h_{2} H t\right)^{c} & q x=1-a+a^{2} / 3 & c=1 / 3 \\
b=1-t\left(-h_{1} H t\right)^{c} & q y=1-b+b^{2} / 3 &
\end{array}
$$

em que $Y=\frac{d}{d a p} ; d=$ diâmetro na altura $h$; altura total; $\mathrm{Tx}=0$ para $\mathrm{d}$ com casca e $\mathrm{Tx}=1$ para $\mathrm{d}$ sem casca; $\beta_{i}=$ parâmetros de regressão, $\operatorname{com} i=0,1, \ldots, 4$; $\varepsilon=$ erro aleatório, sendo $\varepsilon \sim \mathrm{N}\left(0, \sigma^{2}\right)$.

O volume, no modelo de Garay (1979), foi obtido mediante o procedimento de Smalian, utilizando-se os diâmetros estimados pela equação do $d$ desse modelo, para alturas relativas observadas na cubagem. Em uma aplicação prática, recomenda-se adotar seções de 0,1 m para conseguir determinar o volume até um diâmetro preestabelecido. Outra alternativa consiste em estimar a altura $h$ onde o diâmetro $d$ ocorre e, então, estabelecer intervalos em torno de 1,0 m, para estimativa dos diâmetros comerciais e obtenção do volume via Smalian.

As equações ajustadas foram avaliadas por meio do coeficiente de determinação - $R^{2}$, coeficiente de correlação múltipla - $r_{Y Y ̂}$ e análise gráfica dos resíduos. Nos modelos não-lineares, o coeficiente de correlação foi obtido por:

$$
r_{Y \hat{Y}}=\sum_{i=1}^{n} Y_{i} \hat{Y}_{i}-\left(\sum_{i=1}^{n} Y_{i}\right)\left(\sum_{i=1}^{n} \hat{Y}_{i}\right) n^{-1}\left(\sqrt{\left\{\left[\sum_{i=1}^{n}\left(Y_{i}-\bar{Y}\right)^{2}\right]\left[\sum_{i=1}^{n}\left(\hat{Y}_{i}-\bar{Y}\right)^{2}\right]\right\}}\right)
$$

em que:

$$
\begin{aligned}
& \hat{Y}_{i}=\text { diâmetro estimado pela equação; e } \\
& Y_{\mathrm{i}}=\text { diâmetro observado. }
\end{aligned}
$$

Com exceção do modelo de Kozak, os ajustes foram feitos utilizando-se o procedimento Quase Newton, disponível no Software Statistica 5.0.

Além dos modelos de afilamento, foi ajustado um modelo hipsométrico, empregando-se a relação funcional do modelo de Chapman-Richards, da forma:

$$
H t=\alpha\left(1-e^{-\beta d a p}\right)^{(1-\gamma)^{-1}}+\varepsilon
$$

R. Árvore, Viçosa-MG, v.30, n.1, p.99-106, 2006 


\section{RESULTADOS E DISCUSSÃO}

Ao analisar os coeficientes de correlação, entre valores estimados e valores observados, pode-se inferir, em princípio, que os modelos de Kozak e de Garay resultaram em maior precisão. Porém, a variável dependente desses modelos é diferente das variáveis dependentes dos demais (Quadro 1). Além disso, o modelo de Kozak é linear, enquanto os demais são não-lineares. Assim, as estatísticas apresentadas no Quadro 1 servem apenas para fins de comparação relativa com resultados de outros estudos.

Analisando as Figuras 1 e 2, verifica-se que alguns modelos resultam em estimativas inconsistentes de altura comercial, enquanto outros resultam em estimativas menos precisas de diâmetro comercial ou de volume. Isso, de certo modo, é esperado, uma vez que o modelo pode ser bom para estimar uma variável, porém deficiente para outra, ou seja, se um modelo estima $d$ com precisão, não implica estimar $h$ também com precisão. O fato é que se a equação estima o diâmetro ao longo do tronco com precisão e não-tendenciosidade, em geral ele é adequado para estimar volume.

Optou-se pelo modelo de Garay, uma vez que a equação resultante indicou estimativas consistentes das três variáveis implícitas no estudo ( $d, h$ e $V)$, com precisão aceitável (Figuras 1 e 2).

Os coeficientes de correlação entre valores de $d /$ dap observados e estimados foram relativamente baixos em comparação com coeficientes observados na literatura. Isso ocorreu devido à grande amplitude de variação observada nesses valores. Apesar disso, é importante verificar que a tendência de variação de $h / H t$ em relação a $d /$ dap é bem definida (Figura 1).

As equações oriundas dos modelos de Kozak, Demaerschalk e Baldwin resultaram em estimativas inconsistentes de altura comercial (Figura 2). O único modelo que resultou em estimativas precisas e livres de viés foi o de Garay. O próprio Garay (1979) já havia demonstrado a eficiência desse modelo para diferentes espécies. Esse modelo, derivado da função ChapmanRichards, é flexível o suficiente para descrever as variações de forma em árvores de diferentes espécies e tamanhos e consistente em relação às variáveis $d, h$ e $V$.

Para permitir um uso prático da equação obtida a partir do modelo selecionado, foi gerada uma relação hipsométrica utilizando o modelo de Chapman-Richards, sendo a equação resultante apresentada na Figura 3.

A partir do modelo de Garay (Quadro 1), seguese que:

$$
\begin{aligned}
& d \operatorname{dap}^{-1}=\beta_{0}\left(1+\beta_{1} \operatorname{Ln}\left(1-\beta_{2} h^{\beta_{3} H t}{ }^{-\beta_{3}}\right)\right) \\
& d=\operatorname{dap} \beta_{0}\left(1+\beta_{1} \operatorname{Ln}\left(1-\beta_{2} h^{\beta_{3}}{ }^{-\beta^{3}}\right)\right) \mathrm{e} \\
& h=H t\left(\left(-\exp \left(\left(d-\operatorname{dap} \beta_{0}\right)\left(\operatorname{dap} \beta_{0} \beta_{1}\right)^{-1}\right)-1\right) \beta_{2}^{-1}\right)^{1 / \beta_{3}}
\end{aligned}
$$

Substituindo a relação hipsométrica da Figura 3 nessas duas últimas expressões, tem-se, respectivamente:

$$
\begin{aligned}
& d=\operatorname{dap} \beta_{0}\left(1+\beta_{1} \operatorname{Ln}\left(1-\beta_{2}\left(h \alpha^{-1}(1-\exp (-\beta \operatorname{dap}))^{(\gamma-1)^{-1}}\right)^{\beta_{3}}\right)\right) \\
& h=\alpha \exp \left(\left(\operatorname{Ln}\left(-\exp \left(\left(d-\operatorname{dap} \beta_{0}\right)\left(\operatorname{dap} \beta_{0} \beta_{1}\right)^{-1}\right)-1\right)\right.\right. \\
& \left.\left.\beta_{3}^{-1}\right)\right)\left((1-\exp (-\beta \text { dap }))^{(\gamma-1)^{-1}}\right)
\end{aligned}
$$

As expressões 1 e 2 permitem estimar o diâmetro, $d$, em qualquer altura $h$, ou a altura comercial, $h$, para determinado limite de diâmetro comercial, $d$, utilizando como input apenas o dap. Aplicando essas expressões, na faixa de variação de dap observada nos dados foram obtidas as curvas apresentadas na Figura 4. Essa figura contém as variações de forma da espécie estudada e pode ser utilizada para cubar árvores que tiveram apenas o dap medido no campo. O uso desse tipo de gráfico, em ambiente Excel, permite uma melhor identificação do perfil do tronco em árvores de diferentes diâmetros (daps).

Quadro 1 - Coeficientes e estatísticas obtidos através do ajuste de modelos de afilamento para Virola surinamensis Table 1 -Coefficients and statistics obtained through the fitting of taper equations for Virola surinamensis

\begin{tabular}{lccccrc}
\hline \multicolumn{1}{c}{ Modelo } & $\beta_{0}$ & $\beta_{1}$ & $\beta_{2}$ & $\beta_{3}$ & $R^{2}$ & $r_{Y \hat{Y}}$ \\
\hline Kozak & 1,04888 & $-1,78201$ & 1,06724 & - & 0,8216 & 0,9064 \\
Demaerschalk & 0,14374 & 1,04151 & $-0,73679$ & 0,582048 & - & 0,8146 \\
Baldwin & 1,09482 & 0,27786 & - & - & - & 0,8350 \\
Garay1,86696 & 0,14998 & 0,99554 & 0,013315 & - & 0,8472 & \\
\hline
\end{tabular}

R. Árvore, Viçosa-MG, v.30, n.1, p.99-106, 2006 

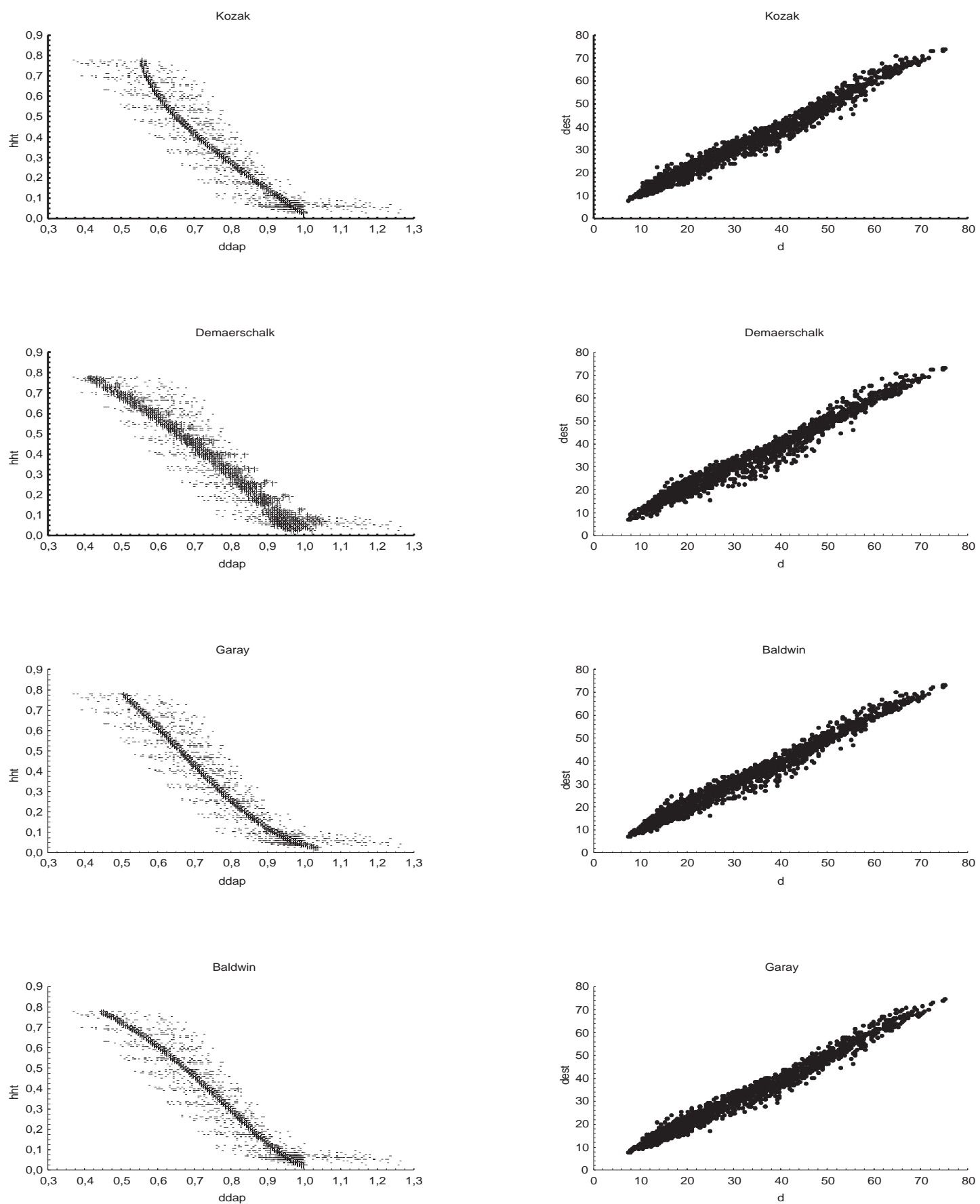

$$
+ \text { estimado observado }
$$

Figura 1 - Perfil do tronco e diâmetros (cm) estimados pelas equações de afilamento versus correspondentes valores observados, utilizando-se os modelos de Kozak, Demaerschalk, Baldwin e Garay.

Figure 1 - Profile and diameters (cm) estimated by the equations versus correspondent observed values, using the Kozak, Demaerschalk, Baldwin and Garay models. 

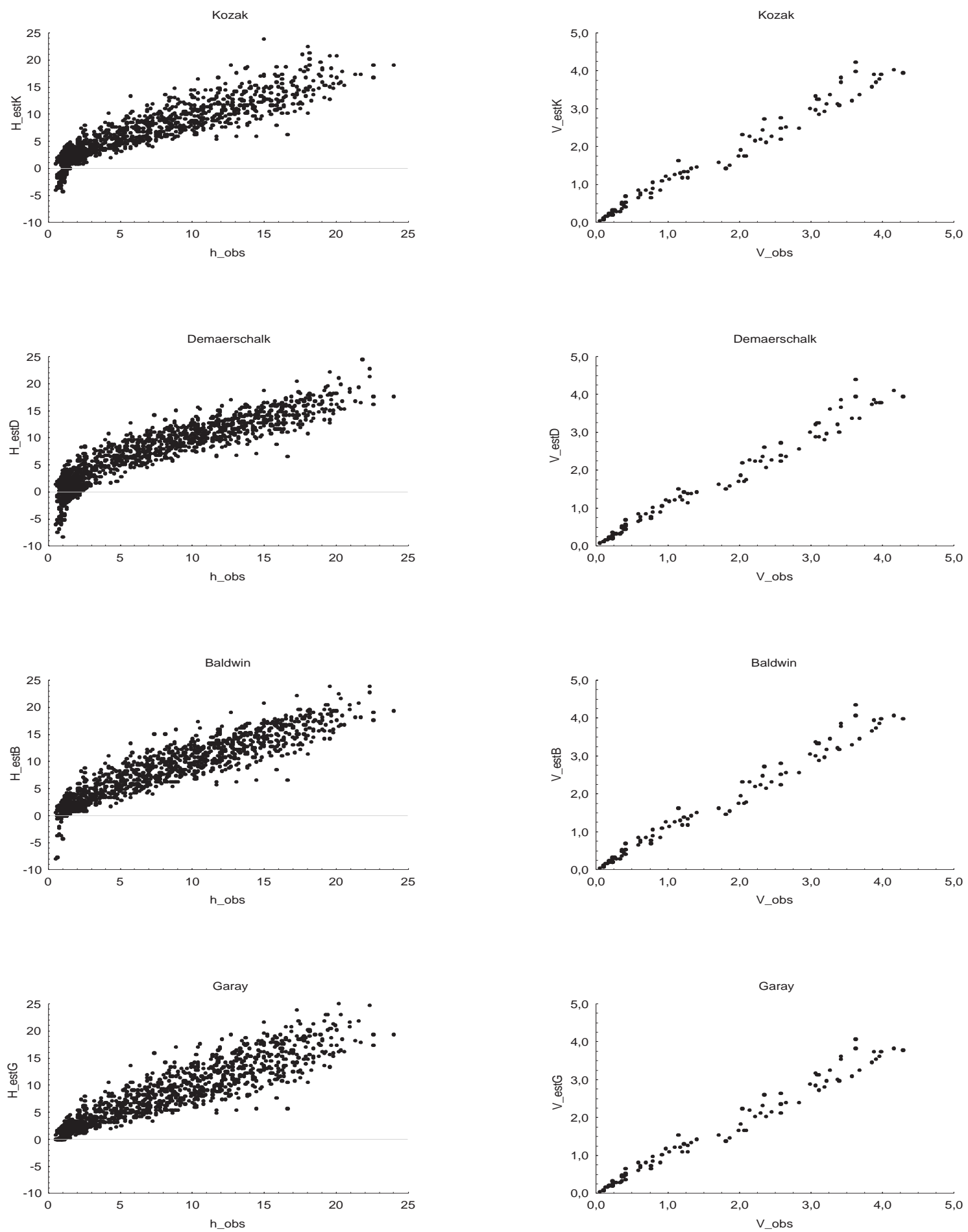

Figura 2 - Altura comercial $(h)$ e volume comercial estimado pelas equações de Kozak, Demaerschalk, Baldwin e Garay versus correspondentes valores observados.

Figure 2 - Commercial height (h) and volume estimated by the Kozak, Demaerschalk, Baldwin and Garay equations versus correspondent observed values.

R. Árvore, Viçosa-MG, v.30, n.1, p.99-106, 2006 


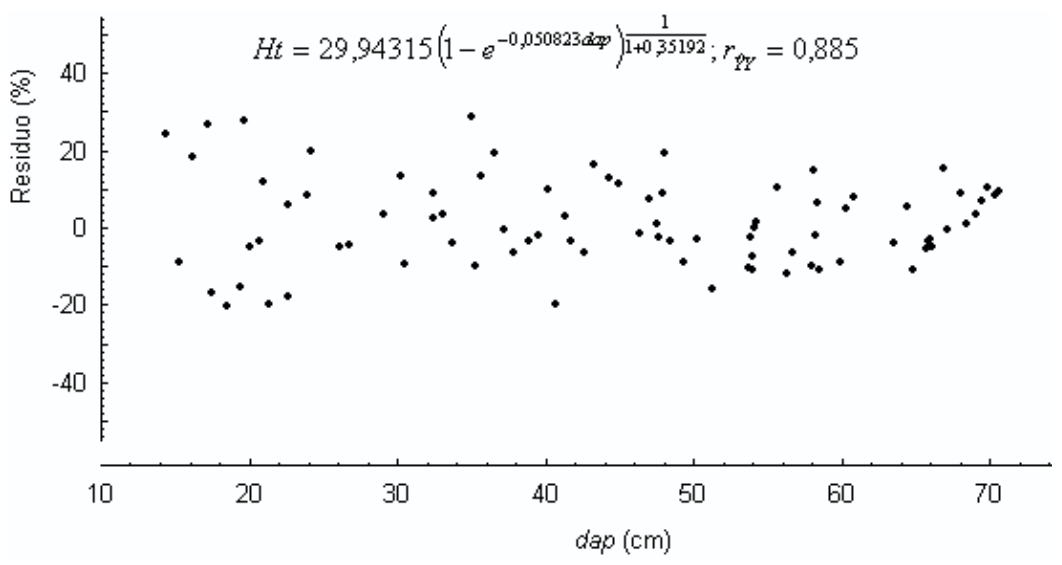

Figura 3 - Resíduos porcentuais das estimativas de altura obtidas a partir do modelo de Richards. Figure 3-Percentage residuals of height estimates obtained from the Chapman-Richards equation.

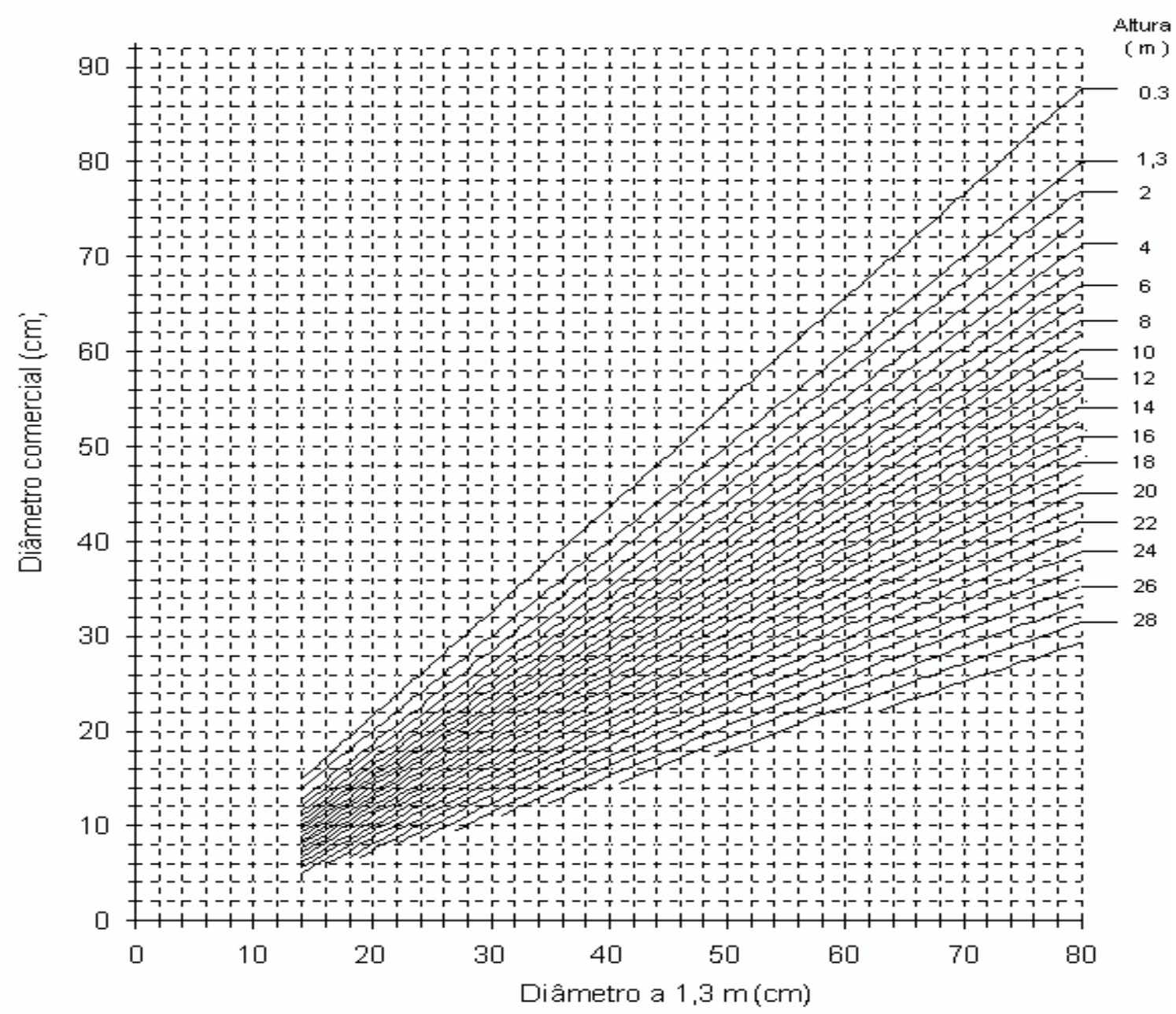

Figura 4 - Relações estimadas entre diâmetro e altura comercial em Virola surinamensis.

Figure 4 - Relations estimated between diameter and commercial height in Virola surinamensis. 


\section{CONCLUSÕES}

A equação:

$d=\operatorname{dap} 1,86696\left(1+0,14998 \operatorname{Ln}\left(1-0,99554 h^{0,013315} H t^{-0,013315}\right)\right)$ obtida a partir do ajuste do modelo de Garay (1979) é indicada para quantificar multiprodutos de árvores de Virola surinamensis. A eficiência de um modelo de afilamento deve ser avaliada com base nas variáveis $d$ e $h$.

\section{REFERÊNCIAS BIBLIOGRÁFICAS}

ANGELO, H.; BRASIL, A. A.; SANTOS, J. Madeiras tropicais: análise econômica das principais espécies florestais exportadas. Acta Amazônica, v. 31, n. 2, p. 237-248, 2001.

BALDWIN JR, V. C.; FEDUCCIA, D. P. Compatible Tree-volume and upper-stem diameter equations for plantation Loblolly Pines in the West Gulf region. South. Journal Applied Forestry, v. 15, n. 2, p. 92-97, 1991.

DEMAERSCHALK, J. P. Converting volume equations to compatible taper equations. Forest Science, v. 18, n. 3, p. 241-245, 1972.

FISCHER, F. Eficiência dos modelos polinomiais e das razões de volume na estimativa volumétrica dos sortimentos e do perfil do fuste de Pinus taeda. 1997. 167f. Dissertação (Mestrado em Engenharia Florestal) - Universidade Federal de Lavras, Lavras, 1997.

GARAY, L. Tropical forest utilization system. VIII. A taper model for entire stem profile including buttressing. Seatlhe. Coll. Forest. Resour., Inst. Forest Prod. Univ. Wash., 1979. 64p. (contrib. 36).

GARCIA, S. L. R.; LEITE, H. G.; YARED, J. A. G. Análise do perfil do tronco de morototó (Didymopamax morototonii) em função do espaçamento. In: CONGRESSO FLORESTAL PANAMERICANO, 1., CONGRESSO FLORESTAL BRASILEIRO, 7., 1993, Curitiba. Anais... Curitiba: SBS/SBEF, 1993. p. 485-491.
GUIMARÃES, D. P.; LEITE, H. G. Um novo modelo para descrever o perfil do tronco. Revista Árvore, v. 16, n. 2, p. 170-180, 1992.

KOZAK, A.; MUNRO, D. D.; SMITH, J. H. G. Taper functions and their application in Forest inventory. Forestry Chronicle, v. 45, n. 4, p. 278-283, 1969.

LOPES, N. P. et al. Circadian and seasonal variation of the essential oil from Virola surinamensis leaves. Phytochemistry, v. 46, n.4, p. 689-983, 1997.

MOURA, J. B. Estudo da forma do fuste e comparação de métodos de estimativa volumétrica de espécies florestais da Amazônia brasileira. 1994. 114f. Dissertação (Mestrado em Engenharia Florestal) Universidade Federal do Paraná, Curitiba, 1994.

OLIVEIRA, N. V. C. Análise florestal da ucuúba numa área de várzea em Ponta de Pedras. Belém: IDESP, 1972. 20 p. (Estudos Paraenses, 37).

RIOS, M. S. A eficiência das funções polinomiais, da função spline cúbica e razões de volume para representar o perfil da árvore e estimar os sortimentos de Pinus elliottii. 1997. 116f. Dissertação (Mestrado em Engenharia Florestal) Universidade Federal de Lavras, Lavras, 1997.

SUDAM / PROJETO DE HIDROLOGIA E CLIMATOLOGIA DA AMAZÔNIA. Atlas Climatológico da Amazônia Brasileira. Belém: 1984. 125 p.

van ROOSMALEN, M. G. M. et al. Frutos da floresta Amazônica. Parte I: Myristicacea. Acta Amazônica, v. 26, n. 4, p. 209-264, 1996.

VIEIRA, L. S. Manual da ciência do solo: com ênfase aos solos tropicais. São Paulo: Agronômica Ceres, 1988. 464p. 